\title{
On Possibility of Space-Charge Compensation in the Fermilab Booster with Multiple Electron Columns
}

\author{
Yu.Alexahin ${ }^{a, 1}$ V. Kapin ${ }^{a, 2}$ \\ ${ }^{a}$ Fermi National Accelerator Laboratory \\ P.O. Box 500, Batavia, IL, USA 60510 \\ E-mail: kapin@fnal.gov
}

ABSTRACT: In this paper we study the possibility of compensation of the space-charge compensation in the Fermilab Booster rapid cycling synchrotron with use several electron columns and show significant promise of such technique.

KEYwORDS: Accelerator modelling and simulations (multi-particle dynamics, single-particle dynamics); Beam dynamics; Beam optics

${ }^{1}$ deceased
${ }^{2}$ corresponding author.




\section{Contents}

1 Introduction $\quad 1$

2 Algorithm 1

3 Results 23

\section{Introduction}

To achieve the Fermilab Accelerator Division Proton Plan goal [1] the number of protons from the Booster should reach $5.25 \cdot 10^{12}$ per batch of 81 bunches. One of the major obstacles on the road to higher intensities is the transverse space charge effect at injection which may lead to fast emittance blowup during bunching.

Using the MAD program, it was shown [2] that a significant (by a factor of 2 or more) transverse emittance blowup may occur in the presence of both space charge and magnetic field errors which break the optics periodicity. The same paper studied the possibility of space charge compensation with a few (1 to 2 ) electron lenses. It turned out that the electron lenses produce an adverse effect on particle dynamics, most likely due to beta-beat excitation.

To reduce the detrimental optics perturbations, it was proposed to use a large number of "electron columns" which can be created by gas jet ionization [3]. The question of how many such "columns" are necessary to produce a positive effect arises. To answer it we performed simplified analysis using the same programs as in study [2] (and even the same distribution of magnetic field errors).

\section{Algorithm}

The "electron columns" were represented by equidistant thin beam-beam elements with equal charges. Their transverse sizes were assumed to coincide with the beam sizes at the particular location as determined by local beta-functions and current values of emittances:

$$
\sigma_{x}=\sqrt{\beta_{x} \varepsilon_{x}+\left(D_{x} \sigma_{p}\right)^{2}}, \quad \sigma_{y}=\sqrt{\beta_{y} \varepsilon_{y}},
$$

where $\sigma_{p}$ is the relative momentum spread ( $\sigma_{p}=0.1 \%$ after bunching) and $D_{x}$ is dispersion function.

The "columns" were placed inside defocusing magnets upstream of long drifts where the betafunctions are approximately equal: $\beta_{x} \approx 13 \mathrm{~m}, \beta_{y} \approx 15 \mathrm{~m}$. Time dependence of the columns' charge followed the density of proton bunch which varies during bunching (Fig.1). To achieve space charge compensation factor $f$ with $n$ columns the charge per column should be

$$
Q_{\text {e.c. }}=-\frac{|e| h N_{b} B_{f}}{n_{\text {columns }}\left(\gamma^{2}-1\right)} f,
$$




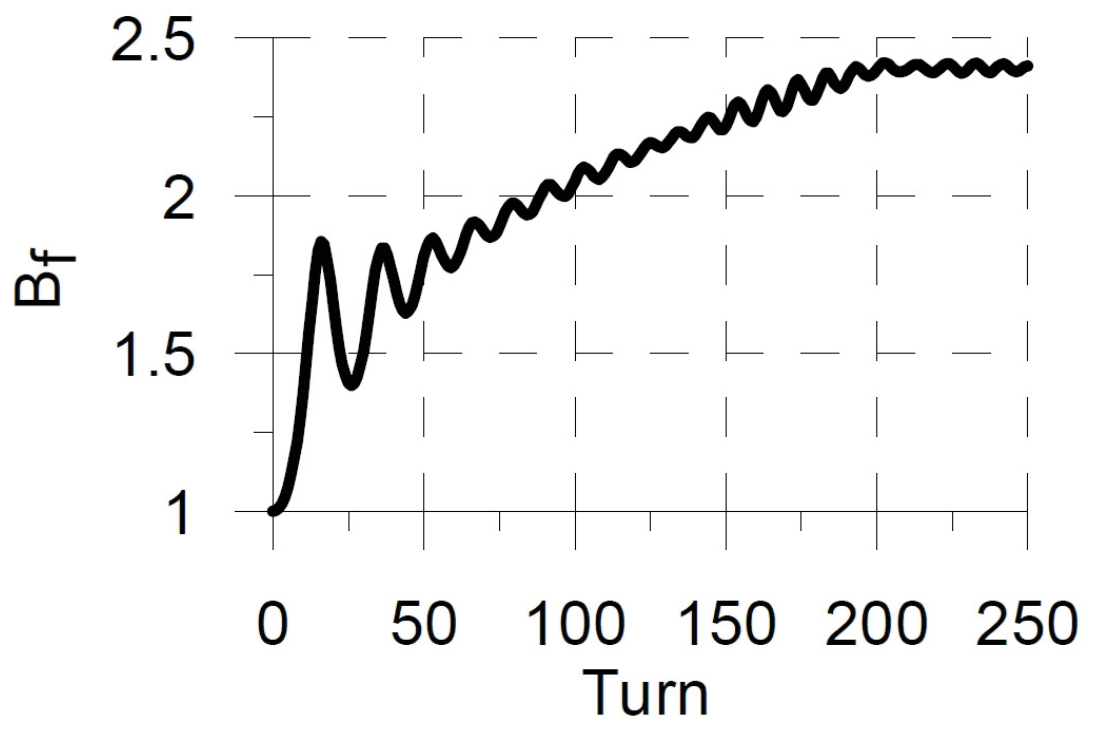

Figure 1. Bunching factor $B_{f}$ vs turn number during the bunching process in Fermilab Booster.

where $h=84$ is the RF harmonic number and $N_{b}$ is the number of protons/bunch.

The space charge of the beam itself can be characterized by the space charge parameter

$$
\xi_{\mathrm{SC}}=\frac{B_{f} r_{p} h N_{b}}{4 \pi \varepsilon_{\perp} \beta^{2} \gamma^{3}},
$$

where $r_{p}=1.54 \cdot 10^{-18} \mathrm{~m}$ is the proton classical radius and $\varepsilon_{\perp}$ is the r.m.s. transverse emittance. Eq. 3 gives the space charge tuneshift in a round beam with no momentum spread. For conditions we consider as nominal for the Proton Plan II - $\varepsilon_{\perp}=1.28 \mathrm{~mm} \cdot \operatorname{mrad}, N_{b}=6 \cdot 10^{10}, \gamma=1.43$ - the space charge parameter is as high as $\xi_{\mathrm{SC}}=0.79$.

The beam space charge was simulated with 197 beam-beam elements distributed around the ring. The charge of an element describing the space charge kick accumulated over distance $L_{k}$ is [2]:

$$
Q_{k}=\frac{|e| h N_{b} B_{f} L_{k}}{\left(\gamma^{2}-1\right) C},
$$

where $C=474.25 \mathrm{~m}$ is the Booster circumference. The simulations were performed using Mathematica and MAD8 according to the following scheme [2]:

- find stationary self-consistent solution for optics with initial $\xi_{\mathrm{SC}}$, store these optics functions for emittance and beam size calculations during tracking (there is no stable optics when $\xi_{\mathrm{SC}}$ crosses the stopband);

- after each turn calculate the action variables from particle positions and momenta at the observation point (end of the lattice) using the stored optics functions;

- find transverse emittances by fitting the obtained distribution in the action variables with Gaussian distribution (exponential in the action variables); 
- calculate the beam sizes from thus found emittances and stored optics functions;

- track particles next turn with the sizes of BEAMBEAM elements (representing both space charge and electron columns) found from the previous turn and the intensity corresponding to the current value of the bunching factor.
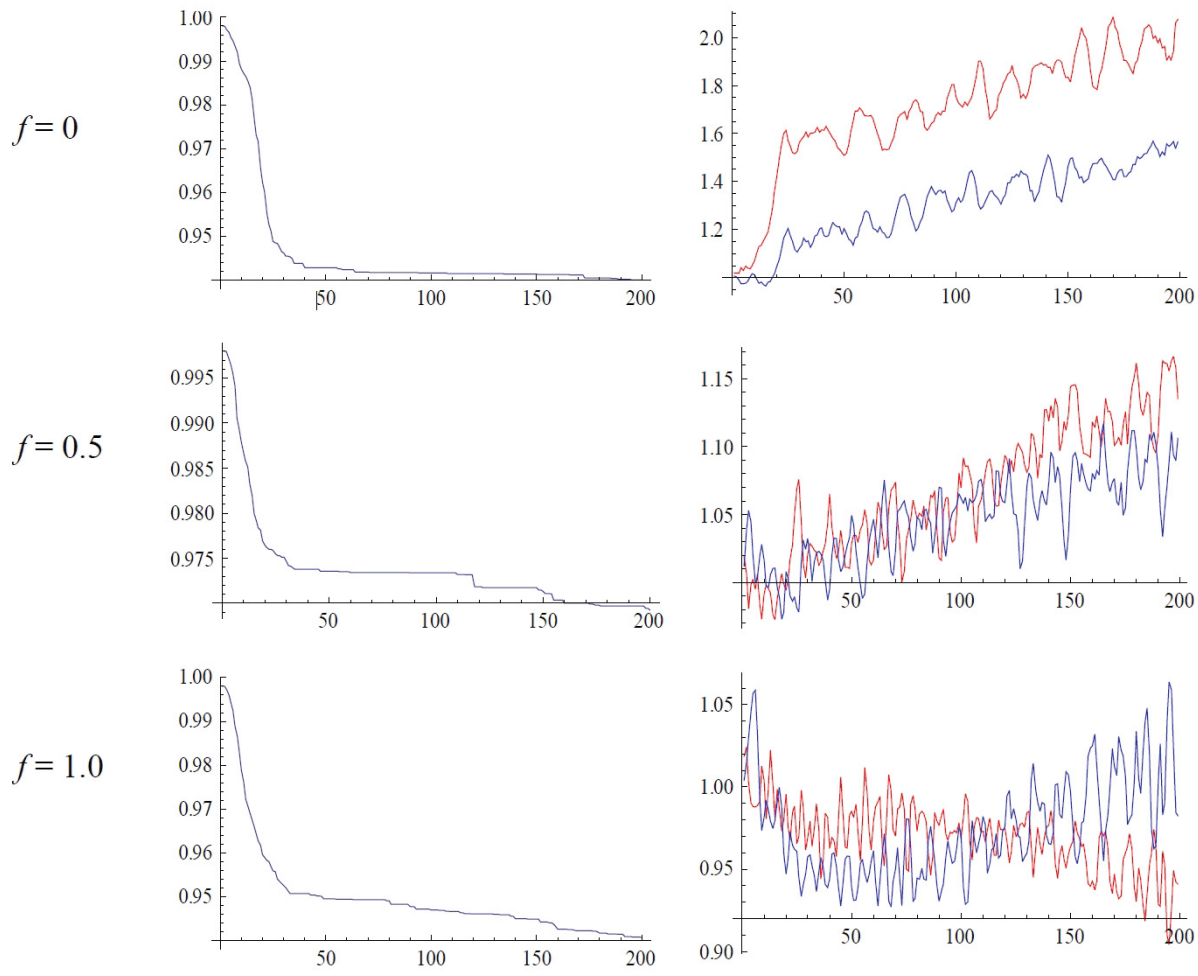

Figure 2. Normalized beam intensity and emittances vs turn number at $N_{b}=6 \cdot 10^{10}, n_{\text {columns }}=24$ and indicated values of the compensation factor $f$.

\section{Results}

Figs.2-5 present tracking results with different bunch intensity $N_{b}$, number of "electron columns" and total compensation factor $f$. Plots in the left columns show normalized beam intensity vs turn number and plots in the right columns show emittances normalized to initial values (red horizontal, blue - vertical).

With "electron columns" placed in each of 24 Booster periods, the space charge compensation drastically reduces emittance blowup and - in the case of increased intensity (Fig.4) - helps the beam to survive at all. It can be seen that even in the case of $f=1$ there is still a noticeable effect of space charge since localized columns provide compensation only on average.

With half as many "columns" (12), the beneficial effect of compensation is less spectacular, even at the same values of the compensation factor $f$ (Figs.3 and 5). Still, it is big enough to pursue this idea. 
It must be noted that the presented results are obtained by 2D simulations; the synchrotron motion was taken into account only as precalculated bunching factor. The space charge gradient modulation along the bunch may introduce synchro-betatron coupling and produce an adverse affect on particle stability.
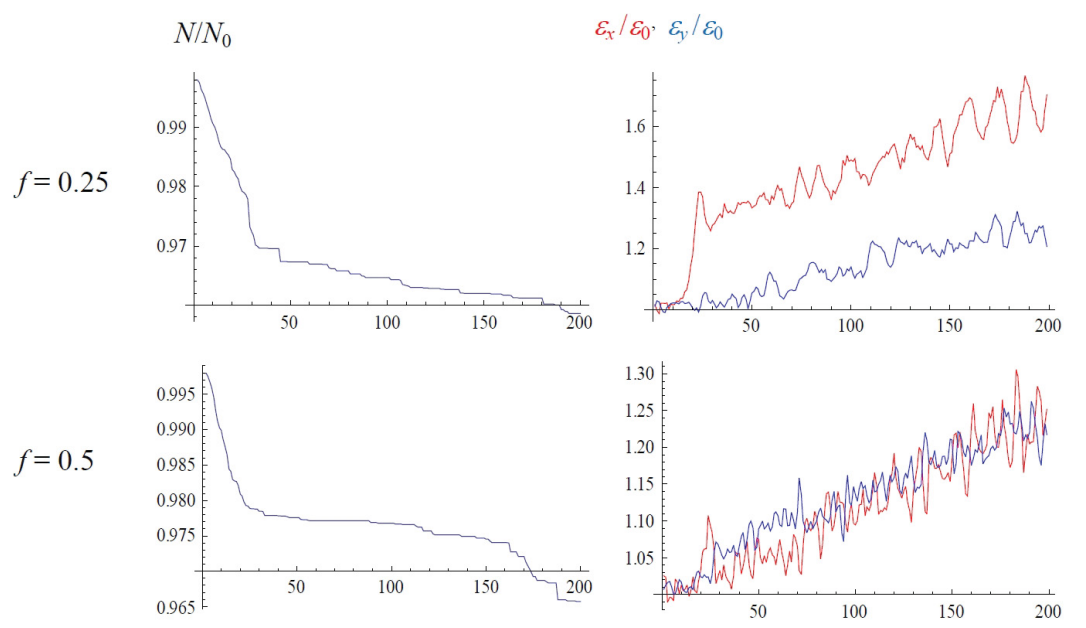

Figure 3. Normalized beam intensity and emittances vs turn number at $N_{b}=6 \cdot 10^{10}, n_{\text {columns }}=12$ and indicated values of the compensation factor $f$.
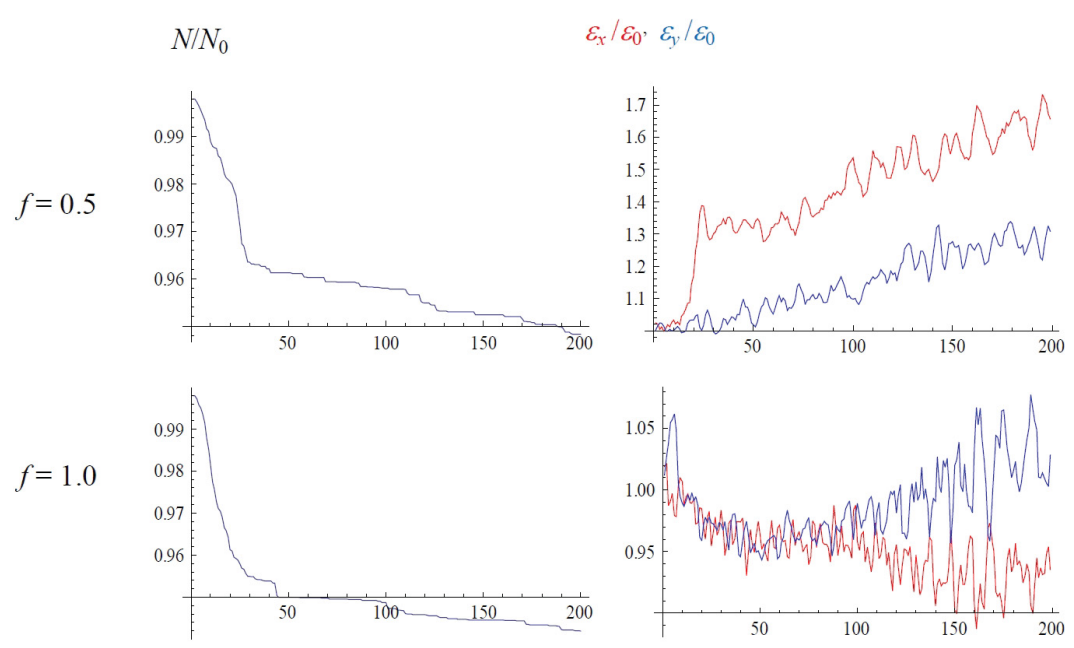

Figure 4. Normalized beam intensity and emittances vs turn number at $N_{b}=9 \cdot 10^{10}, n_{\text {columns }}=24$ and indicated values of the compensation factor $f$. 

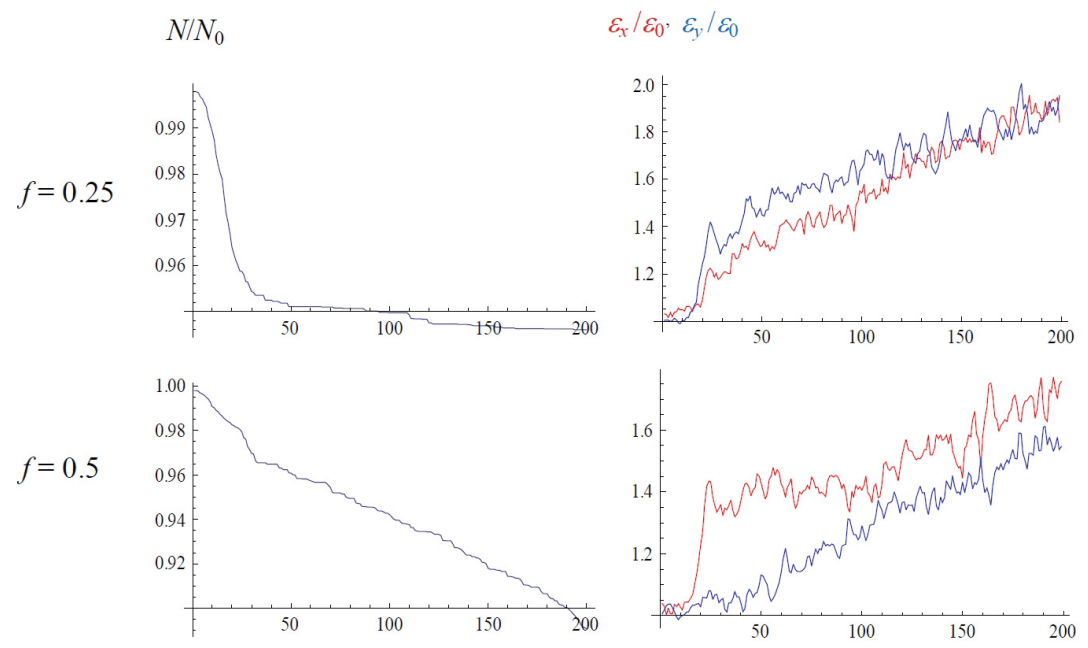

Figure 5. Normalized beam intensity and emittances vs turn number at $N_{b}=9 \cdot 10^{10}, n_{\text {columns }}=12$ and indicated values of the compensation factor $f$.

\section{Acknowledgments}

Authors are grateful to V. Higgins for carefully reading through the manuscript and helpful feedback.

Fermilab is supported by U.S. Department of Energy, Office of Science, Office of High Energy Physics, under Contract No. DE-AC02-07CH11359.

Note added [V.K.(2020)]. This work originally appeared in 2008 as Fermilab internal note beams-doc-3106 (unpublished). The paper was among the first to confirm significant potential improvements due to compensation of space-charge effect by electron lenses or electron column and was often cited as an unpublished article. The first author, now late Yuri Alexahin (1948-2020), greatly valued this first report and always wanted to polish, expand and submit it to a peer-reviewed journal but, as often happens, his plans did not materialize. V.K. took the liberty to slightly reformat the paper for publication in JINST and dedicate it to the memory of his esteemed colleague and very close personal friend.

\section{References}

[1] B. Baller, E.J. Prebys, W.J. Spalding, Proton Plan, Fermilab internal note Beams-doc-1441 (2004, unpublished).

[2] Y. Alexahin, A. Drozhdin, N. Kazarinov, X. Yang, Effects of Space Charge and Magnet Nonlinearities on Beam Dynamics in the Fermilab Booster (Proc. IEEE PAC'07, Albuquerque, New Mexico, USA, 2007), 3474.

[3] V. Shiltsev, New Possibilities for Beam-beam and Space-charge Compensation: MCP Gun and Electron Columns (Proc. IEEE PAC'07, Albuquerque, New Mexico, USA, 2007), 1159. 